Published in final edited form as:

Nat Cell Biol. 2018 September ; 20(9): 1023-1031. doi:10.1038/s41556-018-0146-8.

\title{
FBP17 and CIP4 recruit SHIP2 and Lamellipodin to prime the plasma membrane for Fast Endophilin-Mediated Endocytosis
}

\author{
Laura Chan Wah Hak ${ }^{1,2}$, Shaheen Khan ${ }^{1}$, Ilaria Di Meglio ${ }^{1,3}$, Ah-Lai Law ${ }^{4}$, Safa Lucken- \\ Ardjomande Häsler ${ }^{5}$, Leonor M. Quintaneiro ${ }^{1}$, Antonio P.A. Ferreira ${ }^{1,6}$, Matthias Krause ${ }^{4}$, \\ Harvey T. McMahon ${ }^{5}$, and Emmanuel Boucrot ${ }^{1,7,{ }^{*}}$ \\ ${ }^{1}$ Institute of Structural and Molecular Biology, University College London, Gower Street, London \\ WC1E 6BT, UK \\ ${ }^{4}$ Randall Division of Cell \& Molecular Biophysics, King's College London, New Hunt's House, \\ Guy's Campus, London SE1 1UL, UK \\ ${ }^{5}$ MRC Laboratory of Molecular Biology, Francis Crick Avenue, Cambridge CB2 0QH, UK \\ ${ }^{7}$ Institute of Structural and Molecular Biology, Birkbeck College, Malet Street, London WC1E 7HX, \\ UK
}

\section{Abstract}

Endocytosis mediates the cellular uptake of micronutrients and turnover of plasma membrane proteins. Clathrin-mediated endocytosis (CME) is the major uptake pathway in resting cells1, but several Clathrin-independent endocytic (CIE) routes exist in parallel2,3. One such pathway, fast Endophilin-mediated endocytosis (FEME), is not constitutive but triggered upon activation of certain receptors including $\beta 1$ adrenergic receptor $(\beta 1-\mathrm{AR}) 4$. FEME activates promptly following stimulation as Endophilin is pre-enriched by the $\mathrm{Pi}(3,4) \mathrm{P}_{2}$-binding protein Lamellipodin (Lpd)4,5. However, in the absence of stimulation, Endophilin foci abort and disassemble after a few seconds. Looking for additional proteins involved in FEME, we found that 20 out of 65 BAR domaincontaining proteins tested colocalized with Endophilin spots. Among them, FBP17 and CIP4 prime the membrane of resting cells for FEME by recruiting the 5'-lipid phosphatase SHIP2 and Lpd to mediate local production of $\mathrm{Pi}(3,4) \mathrm{P}_{2}$ and Endophilin pre-enrichment. Membrane-bound GTP-loaded Cdc42 recruits FBP17 and CIP4, before being locally deactivated by RICH1 and

\footnotetext{
*e.boucrot@ucl.ac.uk.

2Present address: Centre for Neural Circuits and Behaviour, University of Oxford, Mansfield Road, Oxford, OX1 3SR, UK

3 Present address: Biochemistry Department, University of Geneva, 30 Quai Ernest Ansermet, Geneva, CH1211, Switzerland

${ }^{6}$ Present address: Department of Biological Chemistry and Molecular Pharmacology, Harvard Medical School, Boston, MA 02115, USA

Reporting Summary. Further information on experimental design is available in the Reporting Summary.

Data availability. The source data for statistical analysis of Figs. 1a-c, 2c, 2d-f, 2h, 3e-f, 3j, 4e, 4g, 5c and 5g are provided in Supplementary Table 2. The mass spectrometry proteomics data have been deposited to the ProteomeXchange Consortium via the PRIDE43 partner repository with the dataset identifier PXD010090 and DOI 10.6019/PXD010090. Uncropped gels and blots are provided in Supplementary Fig. 6. All other data that support the findings of this study are available from the corresponding author upon reasonable request.

Author Contributions

E.B. designed the research and supervised the project. L.C.W.H., S.K., I.D.M. and E.B. performed cell biology experiments. L.C.W.H., S.K., A.L.L, A.P.A.F., E.B. and H.T.McM. performed pull-down experiments. S.L.A.H., I.D.M., S.K. and L.M.Q. provided critical reagents. M.K. helped designing and supervising some experiments. E.B. wrote the manuscript with input from all the other authors.
} 
SH3BP1 GAPs. This generates the transient assembly and disassembly of Endophilin spots, which last 5-10 seconds. This mechanism periodically primes patches of membrane for prompt responses upon FEME activation.

FEME requires the stimulation of cargo receptors by their ligands to trigger the prompt budding of endocytic carriers from the plasma membrane3. FEME carriers are defined as cytoplasmic, Clathrin-negative, Endophilin-positive assemblies (EPAs). The pre-recruitment of Endophilin A (sub-family comprising A1, A2 and A3 in human) into discrete foci on the plasma membrane before receptor activation may enable FEME to be very responsive to cargo activation6. If Endophilin is not pre-enriched, FEME does not take place and cargo receptors either accumulate at the cell surface or enter cells through other alternative pathways3,4. Endophilin binds to the plasma membrane through its Bin1/Amphiphysin/ Rvs161/167 (BAR) domain but is concentrated at endocytic sites by Lamellipodin which itself binds to locally produced $\mathrm{Pi}(3,4) \mathrm{P}_{2} \mathrm{REF} 4,5$. In absence of stimulation, the foci abort and disassemble after few seconds and new ones form nearby (Supplementary Video 1). This is reminiscent to Clathrin-coated pits that frequently abort if they are not stabilized by cargoes and fail to reach a critical size7. However, the spatially localized clustering of Endophilin (prominent at the leading edge) and abortion ahead of FEME activation and endocytic carrier building suggest instead an active mechanism.

In order to identify proteins regulating FEME, we performed pulldown assays using recombinant SH3 domains of Endophilin A1 to 3 (hereafter EndoA1 to 3) and rat brain lysates and identified the binding partners by mass spectrometry (Supplementary Fig. 1a and Table 1). Whilst known interactors such as Dynamin and Synaptojanin were detected, several BAR domain containing proteins were detected. This prompted us to test the binding of the EndoA2 SH3 domain to all BAR proteins containing putative Endophilin-binding proline-rich motifs8 in their primary sequences. We found that Endophilin bound to RICH1 (also called Nadrin-1), SH3BP1, ACAP1, ASAP1, and srGAP1, 2 and 3 but not to the other BAR proteins tested (Supplementary Fig. 1b). The lack of binding of EndoA2 SH3 domain to Pacsin 1-3 (also called Syndapin 1-3) in HEK293 cells extracts suggests potential differences in tissue-specific complexes or post-translational modifications (in particular phosphorylation) between brain and non-neuronal tissues. Together with the previously known binding of Endophilin to Oligophrenin-1REF9 (hereafter OPHN1) and Bin2REF10, this suggested that several BAR domain proteins in addition to Endophilin, could be involved in FEME. A role for several of them during the invagination of EPAs in the absence of a dense protein coat would be coherent with their capacity to sense and induce membrane curvature and functions in local actin nucleation11-13.

To test this, we cloned 65 BAR proteins known in human (Supplementary Fig. 1c and 1a) and tested for their colocalization with endogenous Endophilin foci at the leading edge of resting cells, an area where Endophilin is strongly recruited, but very few clathrin-coated structures are present 4 . We used BSC 1 cells as all but 3 of the BAR proteins tested (FAM92B, Pacsin1 and PSTPIP1) are expressed in kidney, their tissue of origin (Supplementary Fig. 2). Each EGFP-tagged construct was titrated down to achieve low expression and colocalization with endogenous Endophilin staining was measured on 
confocal microscope images using line scans (Supplementary Fig. 3 and 4a-c). To minimize artifactual colocalization, we used the membrane-bound probe CAAX-EGFP as negative control and applied the stringent criterion that both Endophilin and the BAR protein candidate must be enriched on discrete puncta over their surrounding signals (Supplementary Fig. 3b). Therefore any Endophilin foci being located within diffuse BAR protein signals that extend beyond a punctum (such as Arfaptin 1-2, Pick1, RICH2 or GRAF1) were scored negative. Overall, out of the 65 BAR proteins tested, 20 colocalized with high significance ( $\mathrm{p}<0.01$ ) with Endophilin (Fig. 1a and Supplementary Fig. 3 and 4ac). Interestingly, only Amphiphysin, Bin1, Bin2, ICA1-L, RICH1, SH3BP1, OPHN1, ASAP1 and PSTPIP1 were detected on more than 50\% of the foci. FBP17, CIP4, SNX9, Nostrin, Pacsin1 to 3 , and srGAP1 to 3 were detected on a subset of puncta, suggesting that they either marked a subgroup of Endophilin spots or a different timing of recruitment. It is important to note that amongst the BAR proteins identified, only Bin1, RICH1, SH3BP1, ASAP1, FBP17, CIP4, Pacsin2 and srGAP1 and 2 are expressed in most human tissues (Supplementary Fig. 2a), suggesting a potential tissue-specific role for the other candidates. Conscious of the possible artifacts induced by expressing EGFP-tagged transgenes, albeit at lowest possible levels, we validated the colocalization of the candidates for which commercial antibodies were available. We confirmed that endogenous Bin1, CIP4, RICH1, ASAP1, SNX9, Pacsin2 and srGAP1 colocalized to various extent with endogenous Endophilin in BSC1 cells and also in human normal diploid retinal pigmented epithelial (hTERT-RPE1) cells (Fig. 1b-c).

Activation of several receptors including $\beta 1$ adrenergic receptor ( $\beta 1$-AR) triggers FEME4. Stimulation of cells with $\beta 1$-AR agonists induces the prompt $(<10 \mathrm{sec})$ formation of FEME carriers, that contained endogenous $\beta 1-\mathrm{AR}$ but not CME cargoes such as transferrin (Fig. 1c-d and Supplementary Fig. 4d). Following longer incubation times ( $>30 \mathrm{~min})$, endogenous $\beta 1$-AR internalized and accumulated into LAMP-1-positive endosomes in an Endophilindependent but AP2-independent manner (Fig. 1c and Supplementary Fig. 4f), confirming that FEME and not CME was the main endocytic pathway for $\beta 1$-ARREFS4,14. Even though Bin1 and Amphiphysin colocalized on most Endophilin spots at the leading edge, their double knock down (DKD) did not affect $\beta 1$-AR uptake, suggesting that they were either redundant or not involved in the internalization of this particular cargo. By contrast, despite their colocalization on less than 50\% of the Endophilin foci, FBP17 and CIP4 were required for the uptake of $\beta 1$-AR (Fig. 1c-d and Supplementary Fig. 4e). FBP17, CIP4 and TOCA-1 form a sub-group of the F-BAR protein family, having similar domain organization consisting in a F-BAR, HR1 (also called REM) and SH3 domains. We found that, as for several other BAR protein sub-families, FBP17, CIP4 and TOCA-1 can heterodimerize through their BAR domains (Supplementary Fig. 1c-d), suggesting redundancy or cooperation in their functions.

FBP17 and CIP4 are involved in CME and recruited at late stages of clathrin-coated pit formation 15-17, as well as into oscillating waves on the plasma membrane18,19. Their role in $\beta 1$-AR uptake and their clustering into spots on the leading edge of resting cells (Fig. 1b and 2a) suggested an additional role in FEME. 
FBP17 and CIP4 did not bind to $\beta 1$-AR and were not recruited on budded FEME carriers (Fig. 1e and 2b). The inhibition of $\beta 1-A R$ uptake in F+C DKD cells was consistent with the strong reduction $(89.3 \pm 4.7 \%)$ of FEME carrier formation upon receptor activation (Fig. 2c). By contrast, overexpression of FBP17 or CIP4, but not that of TOCA-1, increased the formation of EPAs by over 2-fold upon stimulation (Fig. 2d). These were productive FEME carriers, as measured by receptor interaction with Endophilin (proximity ligation assay, PLA) and $\beta 1$-AR uptake (Fig. 2e-f). The stimulatory effect was PI3K- and Endophilindependent (Fig. 2e-f and Video 3), consistent with the FEME mechanism. The recruitment of Endophilin was strongly reduced $(86.3 \pm 5.2 \%)$ in F+C DKD cells and significantly increased in FBP17 or CIP4-overexpressing cells (Fig. 2c-d). Consistent with their nucleating activity, they were recruited $8 \pm 4 \mathrm{sec}$ before the flashes of Endophilin (Fig. 2g-h and Videos 4 and 5). This explains their low degree of colocalization in fixed samples (Fig. 1a-b). Upon receptor activation, the frequency of spot nucleation increased by over 2 fold but the duration of CIP4 or Endophilin and their order of arrival did not change (Fig. 2h). By contrast, $\beta 1-\mathrm{AR}$ was only recruited to Endophilin spots upon activation. CIP4 overexpression boosted the frequency of spot nucleation and prolonged the spot duration (Fig. 2h), which explains the increase in FEME observed in this condition (Fig. 2d-f). Thus, we concluded that FBP17 and CIP4 were upstream of Endophilin, and were required to recruit it in transient puncta and to prime the membrane of resting cells for FEME.

FBP17 and CIP4 were recently found to bind to SHIP1 and 2 in mast cells and to precede them by $\sim 5 \mathrm{sec}$ on the membrane 19. In fibroblasts, SHIP2 is known to be recruited to the leading edge and to hydrolyse $\mathrm{Pi}(3,4,5) \mathrm{P}_{3}$ into $\mathrm{Pi}(3,4) \mathrm{P}_{2}$ within seconds 20 . We confirmed that FBP17 and CIP4 bound to the ubiquitously expressed SHIP2 and found that they colocalized with it at the leading edge in BSC1 cells (Fig. 3a-b). CIP4 was still recruited to the leading edge in absence of SHIP1 and 2 but not the other way around. Double depletion of SHIP1 and 2 was performed because of the low expression of SHIP1 in kidney (Supplementary Fig. 2b) that could compensate SHIP2 depletion. FBP17 and CIP4 also bound to Lpd and recruited it to the leading edge (Fig. 3a and b). Systematic depletion and overexpression of the various players revealed that FBP17 and CIP4 recruit SHIP2 and Lpd, which then enrich Endophilin at the leading edge of resting cells (Fig. 3d-f). We found that SHIP2 was functionally upstream of $\mathrm{Lpd}$, suggesting that local $\mathrm{Pi}(3,4) \mathrm{P}_{2}$ production was required to stabilize Lpd which is recruited by FBP17 and CIP4. Inhibiting SHIP2 not only strongly reduced the frequency and duration of the priming spots in resting cells, it also blocked the recruitment of $\beta 1$-AR by Endophilin into FEME carriers (Fig. 3h-j and Video 6).

FBP17, CIP4 and TOCA-1 are Cdc42 effectors that bind to the active (GTP-loaded) form through their REM motifs21,22. Inhibiting Cdc42 using ML141 or Secramine or by overexpressing a dominant-negative form was sufficient to strongly reduce endogenous CIP4 recruitment to the leading edges, even in cells depleted for Endophilin (Fig. 4a-e). Inhibiting Cdc42 also hindered the recruitment of SHIP2, Lpd and Endophilin to the leading edge, placing active Cdc42 upstream of them all. CIP4 recruitment to the leading edge was narrowed down to its REM domain (Fig. 4f-h) as only truncated constructs containing this motif were recruited to the leading edge in FBP17+CIP4+TOCA-1 TKD cells (to avoid recruitment of the transgenes by heterodimerization through the BAR domain of endogenous 
proteins). Inhibiting Cdc42 prevented the recruitment of the isolated REM domain. Thus, active Cdc42 recruits FBP17 and CIP4 to prime cells for FEME.

Intriguingly, acute inhibition ( $2 \mathrm{~min}$ ) of $\mathrm{Cdc} 42$ induced the paradoxical hyperactivation of FEME in the first few minutes4, but longer incubation (>10 min) inhibits it (Supplementary Fig. 5a). This suggested that cycles of activation and inhibition of $\mathrm{Cdc} 42$ were required for the transient and periodic recruitment of FBP17 and CIP4 and in foci. Amongst the BAR proteins binding to Endophilin and colocalizing with it at the leading edges are RICH1, SH3BP1 and OPHN1 (Fig. 1a-b and Supplementary Fig. 1-3), which are Cdc42 GAPsREFS23-25. Overexpression of RICH1, SH3BP1 or OPHN1 but not that of their GAP mutated forms inhibited Endophilin recruitment in resting cells, while the depletion of RICH1 and SH3BP1 (R+S DKD) enhanced the phenotype (Fig. 5a-c and Supplementary Fig. 5b). However, CIP4 and Lpd were still recruited to the leading edge of resting $\mathrm{R}+\mathrm{S}$ DKD cells. RICH1 required FBP17 and CIP4 but not Lpd or Endophilin for its recruitment to the leading edge (Fig. 5b and Supplementary Fig. 5c-d). This is consistent with the binding of RICH1, SH3BP1 and OPHN1 to FBP17 (Supplementary Fig. 5e) and CIP4REF24. Akin to CIP4 overexpression, the up-regulation of Endophilin foci by $\mathrm{R}+\mathrm{S}$ DKD primed cells to produce more FEME carriers containing $\beta 1$-AR upon stimulation (Fig. 5d-f). Together, this implies a model whereby a negative feedback loop consisting of local Cdc42 inactivation by GAPs, terminates the local clustering of FBP17/CIP4, SHIP2, Lpd and Endophilin (Fig. 5h). This creates short-lived membrane patches primed to bud FEME carriers that abort in absence of receptor activation.

The finding that discrete patches of the plasma membrane are primed for FEME but rapidly abort and disassemble in absence of receptor stimulation explains the promptness to form endocytic carriers upon activation. When a receptor is activated, it is recruited to pre-existing Endophilin patches that rapidly become FEME carriers. Thus, FEME shares some similarities with CME in the generation of unproductive and abortive events in absence of receptor activation or stabilization7. However, the pre-enrichment mechanism reported here differs from Endophilin recruitment at the latest stages (just before scission) of Clathrincoated pits or IL2R $\beta$-containing vesicles26. It may be that more copies of Endophilin are required during FEME than the 10 to 20 molecules that recruit Dynamin at the neck of forming CME or CIE carriers26,27. The finding that active Cdc42 is required for FEME priming raises the question of potential similarities in the mechanism to that of other Cdc42dependent CIE such as the GRAF1-mediated clathrin-independent carrier (CLIC) pathway, which is also prominent at the leading edges $28-30$ and relying on BAR proteins IRSp53 and PICK131. Even though Cdc42 is involved in both, it acts later during CLIC, localizing onto surface detached carriers and mediating their intracellular maturation32, instead of operating upstream during endocytic protein recruitment.

The early arrival of F-BAR proteins to endocytic sites of shallow curvatures has been reported during $\mathrm{CME}$ where $\mathrm{FCHo} 1$ and 2 prime the pathway33. Mechanistically, the preceding of F-BAR proteins (FBP17 and CIP4) from N-BAR proteins (Endophilin, RICH1 and SH3BP1) is consistent with their preference for different membrane curvatures and membrane tensions 27,34 and possibly aiding their temporal segregation. The presence of multiple N-BAR proteins will likely expand the cargo possibilities of the pathway. 
Strikingly, CIP4, Lpd, SHIP2, SH3BP1 and Endophilin are all overexpressed in various cancers35-39, suggesting a potential role for FEME in tumor formation.

Finally, the potential colocalization of Endophilin with several BAR proteins other than the ones followed up in this study (Fig. 1a), opens the possibility that Endophilin could be recruited by different mechanisms, perhaps in a cell type- or cargo-specific manner to prime cells for FEME. For example, the binding of srGAP3 to Lpd40 suggests an alternative option to Endophilin recruitment to Robo axon guidance receptors or other srGAPdependent receptors. Other BAR proteins are likely to perform additional roles than recruiting Endophilin during FEME.

\section{Methods}

Cell culture

BSC1 (ECACC 85011422) and HEK293 cells (ATCC CRL-1573) were cultured in DMEM (Sigma) supplemented with $10 \%$ fetal bovine serum (FBS, Gold PAA), 1mM GlutaMAX-I (Gibco). Normal human epithelial cells hTERT-RPE1 (ATCC CRL-4000) were cultured in DMEM:F12 HAM (1:1 v/v) (Sigma), 0.25\% sodium bicarbonate (w/v) (Sigma), 1mM GlutaMAX-I (Gibco) and 10\% FBS (Gold, PAA). Approximately $2 \times 10^{6}, 2 \times 10^{5}, 2.5 \times 10^{4}$ or $5 \times 10^{3}$ cells were seeded on $100 \mathrm{~mm}, 35 \mathrm{~mm}$ dishes, $13 \mathrm{~mm}$ coverslips or 96 -well glass bottom plates, respectively. Cells were regularly tested for mycoplasma contamination.

\section{Gene cloning}

Full length and truncated genes (all human, unless specified) were amplified and cloned into pDONR201 (Invitrogen) and transferred into pEGFP, pTagRFP-T (called 'RFP' elsewhere) pMyc or pGEX-6P2 vectors converted into the Gateway system (pDEST vectors made from a pCI backbone), as appropriate: Arfaptin-1 (ARFIP1, IMAGE 6176474), Arfaptin-2 (ARFIP2, IMAGE 2821666), FAM92B (FAM92B, IMAGE8327412), ICA69 (ICA1, cloned from pMWH6 hICA69, a kind gift from H. Kent, MRC LMB), ICA1-like (ICA1L, IMAGE 30377894), PICK1 (PICK1, IMAGE 4026028), Tuba (DNMBP, cloned from pcDNA3TUBA, a kind gift of P. De Camilli, Yale U.), Tuba-L (ARHGEF37, FLJ50067), Amphiphysin-I ( $A M P H I$, IMAGE 5181100), Bin1, also known as Amphiphysin-II (BIN1 iso9, cloned from human brain cDNA library), Bin2-EGFP has been described earlier10, Bin3 (BIN3, IMAGE 3953604), Endophilin-A1 (SH3GL2 iso1, FLJ 92732) full length and SH3 domain (aa 295-end), Endophilin-A2 (SH3GL1, IMAGE 3458016) full length and SH3 domain (aa 311-end), Endophilin-A3 (SH3GL3 iso 1, IMAGE 5197246) full length and SH3 domain (aa 291-end), Endophilin B1 (SH3GLB1, IMAGE1911531) and B2 (SH3GLB2, IMAGE3677306), RICH1, also known as Nadrin-1 ( $A R H G A P 17$, IMAGE 6172310), RICH2, also known as Nadrin-2 ( $A R H G A P 44$, IMAGE 40125967), SH3BP1 (SH3BP1 isoform 1, 100000529), Oligophrenin-1 (OPHN1, cloned from human brain cDNA library), GRAF1b-EGFP ( $A R H G A P 26$ iso $b$, has been described earlier29), GRAF2 ( $A R H G A P 10$, IMAGE 40027832), APPL1 ( $A P P L 1$, IMAGE 4829430), APPL2 (APPL2, IMAGE 5168590), ACAP1, also known as Centaurin- $\beta 1$ ( $A C A P 1$, cloned from pFLAG-CMV2 ACAP1, a gift from Paul Randazzo, NIH), ACAP2, also known as Centaurin- $\beta 2$ ( $A C A P 2$, Kazusa clone KIAA0041), ACAP3, also known as Centaurin- $\beta 5$ (mouse $A C A P 3$, cloned 
from pCI-FLAG ACAP3, a gift from Paul Randazzo, NIH), ASAP1 ( $A S A P 1$, IMAGE 9021132), ASAP2 ( $A S A P 2$, IMAGE 30345039), ASAP3 ( $A S A P 3$, a gift from Tetsuya Takeda, MRC LMB Cambridge), SNX1 ( $S N X 1$, IMAGE 2964409), SNX2 ( $S N X 2$, IMAGE 3048522), SNX4 (SNX4, IMAGE 4641014), SNX5 (SNX5, IMAGE 7939825), SNX6 ( $S N X 6$, IMAGE FLJ77179), SNX7 ( $S N X 7$, IMAGE 4093672), SNX8 ( $S N X 8$, IMAGE 4564502), SNX9 ( $S N X 9$, IMAGE 3832234), SNX18 (SNX18, IMAGE 30341956), SNX30 (mouse SNX30, IMAGE 4950374), SNX32 (SNX32, IMAGE 4797976), SNX33 (SNX33, IMAGE 4869639), FBP17 (rat FNBP1, rapostlin-S short isoform was a kind gift from Manabu Negishi, Kyoto U.) full-length and SH3 domain (aa 549-end), CIP4 (TRIP10 iso2, IMAGE 3532036) full-length, F-BAR (aa 1-264), $\Delta \mathrm{SH} 3$ (aa 1-483), $\triangle \mathrm{F}-\mathrm{BAR}$ (aa 265-545), REM+SH3 (348-545), REM (aa 348-425) and SH3 (aa 483-545) domains, TOCA-1 (FNBP1L, a gift of J. Gallop, U. of Cambridge), Pacsin-1 (PACSIN1, IMAGE 5173129), Pacsin-2 (PACSIN2, IMAGE 2967052), Pacsin-3 (PACSIN3, IMAGE 4302014), PSTPIP1 (PSTPIP1, IMAGE 4180398), PSTPIP2 (PSTPIP2, IMAGE 4524856), Nostrin (NOSTRIN, IMAGE 30327154), GAS7 (GAS7, IMAGE 3353809), FCHSD1, also known as Nwk1 (mouse FCHSD1, IMAGE 6827858, a kind gift from H. Kent, MRC LMB Cambridge), FCHSD2, also known as Nwk2 (FCHSD2, FLJ56467), FCHo1-EGFP and FCHo2-EGFP have been described earlier33, FER ( $F E R$, FLJ96234), FES (FES, IMAGE 5170548), srGAP1 (SRGAP1, IMAGE 6526787), srGAP2 (SRGAP2, IMAGE 40146894), srGAP3 (SRGAP3, cloned from MEGAPa clone, a kind gift from G. Rappold, U. of Heidelberg), HMHA1 (HMHA1, IMAGE 5751491), ARHGAP4 (ARHGAP4, IMAGE 6379390), IRSp53 (BAIAP2, IMAGE 5562784), IRTKS (BAIAP2L1, IMAGE 3842949), Pinkbar (BAIAP2L2, IMAGE 4843302), MIM (MTSS1, DKFZp781P2223), ABBA-1 (MTSS1L, IMAGE 100061592). EGFP-Lpd was described earlier41, EGFP-Cdc42, its dominant-negative (T17N) and constitutively active (Q61L) forms were described earlier42, EGFP-N-WASP was kind gift of Maddy Parsons (King's College London, UK) and EGFP-SHIP2 was a kind gift of Frank Gertler (MIT, USA). The GAP dead mutants of RICH1 (R288A) and RICH2 (R291A) were kind gift of Pontus Aspenstrom (Karolinska Institute) and were subcloned into an EGFP-tagged vector. GAP dead mutants of SH3BP1 (R312A) and OPHN1 (R409A) and by quickchange mutagenesis and sequence verified. The sequences of the DNA primers used for cloning are provided in Supplementary Table 3.

\section{Gene transfection}

For fixed cells colocalization experiments, cells seeded on $13 \mathrm{~mm}$ coverslips (placed in 24well plates) were transfected using Lipofectamine 2000 (Invitrogen) or Nanofectin (PAA) and 0.5 to 500ng DNA depending on the plasmids and the experiments (low or high overexpression). The levels of each plasmid were titrated down to low levels allowing good detection but limiting side effects of overexpression. Cells seeded onto live-cell imaging 35mm glass bottom dishes (MatTek) were transfected using Lipofectamine 2000 (Invitrogen) or Nanofectin (PAA) and 50 to 250ng DNA. For pull-down extracts, HEK293 cells seeded on 100mm dishes were transfected using GeneJuice (Merck) and 1 to $3 \mu \mathrm{g}$ DNA. Cells were incubated $24 \mathrm{~h}$ to express the constructs and were either imaged live, fixed (4\% pre-warmed paraformaldehyde, $20 \mathrm{~min}$ at $37^{\circ} \mathrm{C}$ ) or processed to prepare cell extracts. 
siRNA suppression of gene expression

The siRNA (all Stealth, Invitrogen) used were: Endophilin A1+2+3 TKD: Thermo HSS 109709 (2 oligos against human SH3GL2), Thermo HSS109707 (2 oligos against SH3GL1), Thermo HSS109712 (2 oligos against human SH3GL3); FBP17 set 1: Thermo HSS118093 (2 oligos against human FNBP1), set 2: Thermo HSS118094 (2 oligos against human FNBP1), set 3: Thermo HSS118095 (2 oligos against human FNBP1); CIP4 set 1: Thermo HSS113814 (2 oligos against human TRIP10), set 2: Thermo HSS190195 (2 oligos against human TRIP10), set 3: Thermo HSS113813 (2 oligos against human TRIP10); TOCA-1 set 1: Thermo HSS123422 (2 oligos against human FNBP1L), set 2: Thermo HSS123421 (2 oligos against human FNBP1L), set 3: Thermo HSS123423 (2 oligos against human FNBP1L). Double knock-down (DKD) of FBP17+CIP4 and triple knock-down (TKD) FBP17+CIP4+TOCA-1 were achieved by combining the oligos from the respective set 1. Lamellipodin: Dharmacon ON-TARGETplus SMARTpool (mix of J-031919-08, J-031919-07, J-031919-06 and J-031919-05 targeting human RAPH1) or Thermo HSS127957 (2 oligos against human RAPH); SHIP1: Dharmacon ON-TARGETplus SMARTpool (mix of J-003013-09, J-003013-10, J-003013-11 and J-003013-12 targeting human INPP5D); SHIP2: Dharmacon ON-TARGETplus SMARTpool (mix of J-004152-06, J-004152-07, J-004152-08 and J-004152-09 targeting human INPPL1); RICH1: Thermo HSS123922 (2 oligos against human ARHGAP17); RICH2: Thermo HSS114994 (2 oligos against human ARHGAP44); SH3BP1: Thermo HSS119062 (2 oligos against human SH3BPI); GRAF1: Dharmacon ON-TARGETplus SMARTpool (mix of WILDE-000564 and WILDE-000531 targeting human ARHGAP26); GRAF2: Thermo HSS128606 (2 oligos against human ARHGAP10); OPHN1: Thermo HSS107440 (2 oligos against human OPHN1); AP2: HSS101955 (2 oligos against human AP2M1). Control siRNA used were Dharmacon ON-TARGETplus GAPDH control (D-001830-01-05), Invitrogen Stealth control (scrambled) oligo 138782 or a mixture of Invitrogen Stealth 'high GC' 45-2000 and 'low GC' 45-2002 oligos. The sequences of the siRNA primers are provided in Supplementary Table 3. Cells seeded on $13 \mathrm{~mm}$ coverslips placed in 24 well plates were transfected twice (on day 1 and 2) with Oligofectamine (Invitrogen) complexed with 16 pmol of each indicated siRNA and analyzed 3-4 days after the first transfection. EGFPtagged CIP4 domains targeting was performed with plasmid DNA transfection of the indicated constructs at the middle of day 3 (when endogenous levels of the targeted proteins were already decreased) and the cells were analyzed 16h later (on day 4). RNAi knock-down efficiency was verified by western-blotting and immunofluorescence counter-staining. The use of validated pools of siRNA targeting the same genes increased the knock-down efficiency and specificity.

\section{Expressed sequence tag (EST) tissue abundance}

Data were recorded from the NCBI UniGene transcriptome database using the following entries: ARFIP1, Hs.416089; ARFIP2, Hs.75139; FAM92B, Hs.125713; ICA1, Hs.487561; ICA1L, Hs.516629; PICK1, Hs.180871; DNMBP, Hs.500771; ARHGEF37, Hs.256206; AMPHI, Hs.592182; BIN1, Hs.193163; BIN2, Hs.14770; BIN3, Hs.433722; SH3GL1, Hs. 97616; SH3GL2, Hs.75149; SH3GL3, Hs.270055; SH3GLB1, Hs.136309; SH3GLB2, Hs. 460238; ARHGAP17, Hs.373793; ARHGAP44, Hs.499758; SH3BP1, Hs.601143; OPHN1, Hs.128824; ARHGAP26, Hs.654668; ARHGAP10, Hs.368631; APPL1, Hs.476415; 
APPL2, Hs.506603; ACAP1, Hs.337242; ACAP2, Hs.593373; ACAP3, Hs.535257; ASAP1, Hs.655552; ASAP2, Hs.555902; ASAP3, Hs.437379; SNX1, Hs.188634; SNX2, Hs. 713554; $S N X 4$, Hs.507243; SNX5, Hs.316890; SNX6, Hs.356647; SNX7, Hs.197015; SNX8, Hs.584900; $S N X$ 9, Hs.191213; SNX18, Hs.432755; SNX30, Hs.522350; SNX32, Hs.591950; SNX33, Hs.8705; FNBP1, Hs.189409; TRIP10, Hs.515094; FNBP1L, Hs. 515094; PACSIN1, Hs.520087; PACSIN2, Hs.162877; PACSIN3, Hs.334639; PSTPIP1, Hs. 129758; PSTPIP2, Hs.567384; NOSTRIN, Hs.189780; GAS7, Hs.462214; FCHSD1, Hs. 591257; FCHSD2, Hs.744959; FCHO1, Hs.96485; FCHO2, Hs.165762; FER, Hs.221472; FES, Hs.7636; SRGAP1, Hs.210751; SRGAP2, Hs.497575; SRGAP3, Hs.654743; HMHA1, Hs.465521; ARHGAP4, Hs.701324; BAIAP2, Hs.128316; BAIAP2L1, Hs. 656063; BAIAP2L2, Hs.474822; MTSS1, Hs.336994; MTSS1L, Hs.432387; RAPH1, Hs. 471162; SHIP1, Hs.262886; SHIP2, Hs.523875; GAPDH, Hs.544577.

\section{Protein purification and pull down experiments}

GST-tagged SH3 domains were expressed in BL21-codonPlus(DE3)-RP E.coli (Stratagene). Cells were lysed using either by sonication or using Emulsiflex C3, spun at 18,000 to $140,000 \mathrm{~g}$ for 20 to $40 \mathrm{~min}$ (depending on the construct) at $4^{\circ} \mathrm{C}$ and the supernatants were bound to glutathione beads for $30 \mathrm{~min}$ to $1 \mathrm{~h}$. The beads were washed extensively with $150 \mathrm{mM} \mathrm{NaCl}$, 20mM HEPES pH 7.4, 2mM DTT, 2mM EDTA, including 2 washes with $500 \mathrm{mM} \mathrm{NaCl}$ or five times with ice cold PBS. GST-proteins were eluted from the GSTsepharose beads with $10 \mathrm{mM}$ glutathione, further purified by Superdex 200 gel filtration, and rebound to a minimal volume of fresh GST-sepharose beads (to achieve saturation) for use in pull downs. Brain lysates $(5-10 \mathrm{mg} / \mathrm{mL})$ were prepared in lysis buffer $(150 \mathrm{mM} \mathrm{NaCl}, 20 \mathrm{mM}$ HEPES, 5mM DTT, $0.1 \%$ Triton X-100 and a protease and phosphatase inhibitor cocktail (Thermo Scientific)). Bead-bound proteins were then exposed to rat brain or cell lysates for $30 \mathrm{~min}$ to $1 \mathrm{~h}$ on ice, pelleted in a cooled desktop centrifuge, and washed three times in lysis buffer. The final pellet was boiled in sample buffer and ran on SDS-PAGE. For protein identification, Coomassie Brilliant Blue-stained protein bands were excised from the gels and underwent LC-MS-MS mass spectrometry (Thermo Orbitrap) for identification. For candidate validation, HEK293 cells expressing various EGFP-tagged constructs were quickly washed with cold PBS, lysed in ice-cold lysis buffer (as above or 50mM Tris HCL, $200 \mathrm{mM} \mathrm{NaCl}, 1 \% \mathrm{NP}-40,2 \mathrm{mM} \mathrm{MgCl} 2,10 \%$ glycerol, $\mathrm{pH} 7.4,1 \mathrm{mM} \mathrm{Na}_{3} \mathrm{VO}_{4}, 10 \mathrm{mM} \mathrm{NaF}$, and protease inhibitors 'Complete mini without EDTA', Roche), briefly sonicated and spun at 14,000 to $17,000 \mathrm{~g}$ for 10 to $15 \mathrm{~min}$ at $4^{\circ} \mathrm{C}$. Protein concentration was then determined (Pierce BCA protein assay kit; Thermo Fisher Scientific). Bead bound proteins were then incubated with cell lysates for 1 to $2 \mathrm{~h}$, pelleted in a cooled benchtop centrifuge, and washed three times in lysis buffer. The final pellets were boiled in sample buffer and ran on SDSPAGE ('input' lanes correspond to 1 to $10 \%$ of cell extracts). The proteins were transferred onto PVDF membrane and immunoblotted using anti-EGFP antibodies, followed by HRPcoupled secondary antibodies (BioRad or Dako). Blots were developed with the ECL kit (Thermo Fischer Scientific or Merck Millipore) and x-ray film, or the Immun-Star WesternC ECL kit (Bio-Rad Laboratories) using the Bio-Rad Imager and ImageLab software. 


\section{Co-immunoprecipitations}

RPE1 or HEK293 cells were co-transfected with equal amounts of Myc- and EGFP-tagged

BAR domain constructs. After 16-24h expression, cells were quickly washed with cold PBS, lysed in ice-cold lysis buffer (10mM Tris HCL pH7.5, 150mM NaCl, 0.5mM EDTA, 0.5\% NP40 and a protease and phosphatase inhibitor cocktail (Thermo Scientific)) and spun at $14,000 \mathrm{~g}$ for $10 \mathrm{~min}$ at $4^{\circ} \mathrm{C}$. Cell lysates were incubated with GFP-TRAP_A (Chromotek) bead slurry for up to $16 \mathrm{~h}$ at $4^{\circ} \mathrm{C}$. The beads were washed 3 times (10mM Tris HCL pH7.5, $150 \mathrm{mM} \mathrm{NaCl}, 0.5 \mathrm{mM}$ EDTA). The final pellets and unbound fractions were boiled in sample buffer and ran on SDS-PAGE ('input' lanes correspond to 1 to $10 \%$ of cell extracts). The proteins were transferred onto PVDF membrane and immunoblotted using anti-Myc or anti-EGFP antibodies, followed by HRP-coupled secondary antibodies (BioRad). Blots were developed with the ECL kit (Thermo Fischer Scientific or Merck Millipore) and X-ray films.

\section{Chemicals and small inhibitors}

Dobutamine ( $\beta 1$ adrenergic receptor partial agonist, Sigma, D0676) was used at $10 \mu \mathrm{M}$,

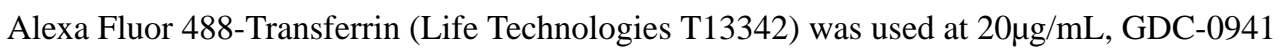
(called 'PI3Ki' in this study, Symansis SYG0941) was used at 5 to 20nM; AS19499490

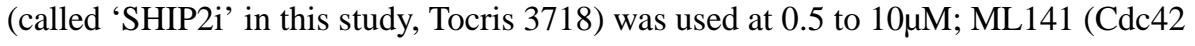
allosteric inhibitor, called 'CDC42i 1' in this study, Tocris 4266) was used at $10 \mu \mathrm{M}$ and secramine (Cdc42 inhibitor, called 'CDC42i 2' in this study, was a kind gift from T. Kirchhausen (Harvard Medical School) and the Hammond lab (U. of Louisville) and was synthetized by Bo Xu and GB Hammond and was used at $10 \mu \mathrm{M}$.

\section{Cell stimulation and cargo uptake}

Cells were never serum-starved or pre-incubated at $4{ }^{\circ} \mathrm{C}$. 'Resting' conditions correspond to cells being cultured in $10 \%$ serum media and directly fixed. $\beta 1$ adrenergic receptor stimulation (which activates FEME) was performed by incubating cells at $37^{\circ} \mathrm{C}$ for $30 \mathrm{sec}$ to $30 \mathrm{~min}$ with pre-warmed medium containing $10 \mu \mathrm{M}$ dobutamine. EGF stimulation was performed by incubating cells at $37^{\circ} \mathrm{C}$ for $30 \mathrm{sec}$ with pre-warmed medium containing $50 \mathrm{ng} / \mathrm{mL}$ EGF. In some experiments, cells were incubated with pre-warmed medium containing 50 $\mathrm{g} / \mathrm{mL}$ Alexa Fluor-488 labeled human transferrin (CME cargo) and $10 \mu \mathrm{M}$ dobutamine at $37^{\circ} \mathrm{C}$ for 30 seconds. After the incubation periods at $37^{\circ} \mathrm{C}$, cells stimulated as described above were quickly washed once with $37^{\circ} \mathrm{C}$ pre-warmed PBS to removed unbound ligands and fixed with pre-warmed $4 \%$ PFA for $20 \mathrm{~min}$ at $37^{\circ} \mathrm{C}$. Fixed cells were then washed three times with PBS and one time with PBS supplemented with $50 \mathrm{mM} \mathrm{NH}_{4} \mathrm{Cl}$ to quench free PFA. Cells were then permeabilized ( $0.05 \%$ saponin), immunostained and imaged as described below.

\section{Antibodies}

The following antibodies were used for immunostaining or immunoblotting: anti-Endophilin A2 clones H-60 and A-11 (rabbit polyclonal sc-25495 and mouse monoclonal sc-365704, respectively, Santa Cruz Biotechnology), anti- $\beta 1$ adrenergic receptor (rabbit polyclonal, AbCam ab3442 for immunostaining or Thermo Scientific PA1-049 for western-blotting), anti-Lamellipodin (Lpd) (mouse monoclonal clone H-5, Santa Cruz sc-390050 and rabbit 
polyclonal, Atlas Antibodies HPA020027), anti-CIP4, (mouse monoclonal clone 21, Santa Cruz sc-135868), anti-Bin1 (rabbit polyclonal, GeneTex GTX103259), anti-RICH1 (rabbit polyclonal, Bethyl Labs A304-689A), anti-SNX9 (mouse monoclonal clone 2F1, AbCam ab118996), anti-Pacsin2 (rabbit polyclonal, Abgent AP8088b), anti-srGAP1 (mouse monoclonal clone 5D2, Abnova H00057522-M03), anti-ASAP1 (mouse monoclonal clone P7Q, Santa Cruz sc-81896), anti-SHIP2 (mouse monoclonal clone 3E6, Novus Biological H00003636-M01 or rabbit monoclonal clone C76A7, Cell Signaling Technologies 2839), anti-a-adaptin clone 8 (mouse monoclonal, BD Bioscience 610501), anti-GAPDH clone 14C10 (rabbit polyclonal, Cell Signaling Technologies 2118), anti-LAMP-1 (mouse monoclonal clone H4A3-c, Developmental Studies Hybridoma Bank or rabbit polyclonal D2D11, Cell Signaling Technologies 9091), anti-EGFP (rabbit polyclonal, AbCam ab290 or mouse monoclonal clones 7.1 and 13.1, Roche 11814460001), anti-Myc tag clone 71D10 (rabbit monoclonal, Cell Signaling Technologies 2278). Primary antibodies were used at 0.1 to $1 \mu \mathrm{g} / \mathrm{mL}$ for immunoblotting and 0.1 to $2 \mu \mathrm{g} / \mathrm{mL}$ for immunofluorescence. The following secondary antibodies were used: AlexaFluor 488 (A-11001) and AlexaFluor 555 (A-21422) Goat anti-Mouse IgG, AlexaFluor 488 (A-11008) and AlexaFluor 555 (A-21428) Goat antiRabbit IgG (all from Thermo Scientific) and Goat anti-Mouse IgG-HRP conjugate (1706516) and Goat anti-Rabbit IgG-HRP conjugate (1706516) from Bio-Rad. See Supplementary Table 4 for full antibody details.

\section{Immunostaining, proximity ligation assays, laser scanning confocal fluorescent microscopy and analysis}

Fixed samples were permeabilized ( $0.05 \%$ saponin) and immunostained (primary and secondary antibodies were diluted in 5\% BSA). In some experiments, Phalloidin-AlexaFluor 647 (Cell Signaling Technologies 8940) was added with secondary antibodies. Proximity ligation assays (PLA) were performed using Duolink PLA technology (kit DUO92101, Sigma), following instructions given by the manufacturer. The coverslips were mounted with DABCO anti-fade agent on glass slides and imaged using a laser scanning confocal microscope (TCS Sp5 AOBS; Leica) equipped with a 63x objective. For Alexa488, the illumination was at 488nm and emission collected between 498 and 548nm; for Alexa555 the laser illumination was at $543 \mathrm{~nm}$ and emission collected between 555 and $620 \mathrm{~nm}$; for Alexa647 and DRAQ5, the laser illumination was at 633nm and emission collected between 660 and 746nm. The percentages of colocalization of BAR proteins with Endophilin spots located at the leading edge of cells were determined using Volocity 6.0. The levels of CIP4 EGFP-tagged constructs at the leading edges of cells were measured on masks covering a total at least $100 \mu \mathrm{m}$ long ( $2 \mu \mathrm{m}$ wide) of leading edge of at least 3 cells from 3 independent experiments using Volocity 6.0.

\section{Live-cell confocal fluorescent microscopy}

Just before live-cell imaging, the medium of cells grown on MatTek dishes was changed to a-MEM without phenol red, supplemented with 20mM HEPES, pH 7.4 and 5\% FBS and placed into a temperature controlled chamber on the microscope stage with $95 \%$ air: $5 \%$ $\mathrm{CO}_{2}$ and $100 \%$ humidity. Live-cell imaging data were acquired using a fully motorized inverted microscope (Eclipse TE-2000, Nikon) equipped with a CSU-X1 spinning disk confocal head (UltraVIEW VoX, Perkin-Elmer, England) using a 60x lens (Plan 
Apochromat VC, 1.4 NA, Nikon) under control of Volocity 6.0 (Improvision, England). 14bit digital images were obtained with a cooled EMCCD camera (9100-02, Hamamatsu, Japan). Four 50mW solid-state lasers (405, 488, 561 and 647nm; Crystal Laser and Melles Griots) coupled to individual acoustic-optical tunable filter (AOTF) were used as light source to excite EGFP, Alexa488, TagRFP-T, Alexa555 and 647, as appropriate. Rapid twocolour time-lapses were acquired at 500ms to $2 \mathrm{~s}$ intervals, using a dual (525/50; 640/120, Chroma) emission filter respectively. The power of the lasers supported excitation times of $50 \mathrm{~ms}$ in each wavelength and the AOTFs allowed minimum delay ( 1ms) between 2 colors (e.g. delay between green-red for each timepoint), which was an important factor to assess the colocalization between markers.

\section{Plasma membrane isolation}

Cell surface protein isolation, excluding intracellular proteins, was performed as in 4 , by selective biotinylation using a cell-impermeable cleavable biotinylation reagent (Sulfo-NHSSS-Biotin), followed by purification of surface proteins by affinity-purified NeutrAvidin agarose resin (Thermo Scientific 89881).

\section{Statistics and reproducibility}

The samples sizes and statistical tests were selected based on previous studies with similar methodologies. Sample sizes were not determined using statistical methods. All experiments were independently repeated at least three times, giving similar results. For all figures, results shown are mean \pm standard error of the mean (SEM). Statistical testing was performed using Prism 6 (GraphPad Software). Comparisons of data in Fig. 1a, 2e, 2f, 3j middle, $3 \mathrm{j}$ right, $4 \mathrm{~g}$, $5 \mathrm{c}$ and $5 \mathrm{~g}$ middle were performed by one-way analysis of variance (ANOVA) with Tukey's multiple comparison test. Comparisons of all other data were performed by two way ANOVA with Tukey's multiple comparisons test. $P$ values $<0.01$ were considered significant.

\section{Supplementary Material}

Refer to Web version on PubMed Central for supplementary material.

\section{Acknowledgments}

We thank Sew Yew Peak-Chew for mass spectrometry, Mina Edwards, Maud Dumoux and Kieran McGourty for technical help, Pietro De Camilli (Yale U.), Pontus Aspenstrom (Karolinska Institute), Paul Randazzo (NIH), Manabu Negishi (Kyoto U.), Gudrun Rappold (U. of Heidelberg), Helen Kent (MRC LMB Cambridge), Jennifer Gallop (U. of Cambridge), Tetsuya Takeda (MRC LMB Cambridge), Maddy Parsons (King's College London, UK), Frank Gertler (MIT, USA) and Tomas Kirchhausen (Harvard Medical School) for the kind gift of reagents and the members of the Boucrot lab for helpful comments. E.B. was a Biotechnology and Biological Sciences Research Council (BBSRC) David Phillips Research Fellow (BB/R01551X/1), a Lister Institute Research Fellow and a recipient of a grant from the Royal Society Research Grant (RG120481). S.L-A.H. and H.McM. were supported by the Medical Research Council UK (grant number U105178805 to H.McM.) and by the Swiss National Science Foundation [fellowship number PA00P3-124164 to S.L-A.H]. A.P.A.F was supported by the Fundação para a Ciência e Tecnologia.

\section{References}

1. Bitsikas V, Correa IR Jr, Nichols BJ. Clathrin-independent pathways do not contribute significantly to endocytic flux. Elife. 2014; 3:e03970. [PubMed: 25232658] 
2. Johannes L, Parton RG, Bassereau P, Mayor S. Building endocytic pits without clathrin. Nat Rev Mol Cell Biol. 2015; 16:311-321. [PubMed: 25857812]

3. Ferreira APA, Boucrot E. Mechanisms of Carrier Formation during Clathrin-Independent Endocytosis. Trends Cell Biol. 2018; 28:188-200. [PubMed: 29241687]

4. Boucrot E, et al. Endophilin marks and controls a clathrin-independent endocytic pathway. Nature. 2015; 517:460-465. [PubMed: 25517094]

5. Vehlow A, et al. Endophilin, Lamellipodin, and Mena cooperate to regulate F-actin-dependent EGFreceptor endocytosis. EMBO J. 2013; 32:2722-2734. [PubMed: 24076656]

6. Watanabe S, Boucrot E. Fast and ultrafast endocytosis. Curr Opin Cell Biol. 2017; 47:64-71. [PubMed: 28391090]

7. Ehrlich M, et al. Endocytosis by random initiation and stabilization of clathrin-coated pits. Cell. 2004; 118:591-605. [PubMed: 15339664]

8. Cestra G, et al. The SH3 domains of endophilin and amphiphysin bind to the proline-rich region of synaptojanin 1 at distinct sites that display an unconventional binding specificity. J Biol Chem. 1999; 274:32001-32007. [PubMed: 10542231]

9. Nakano-Kobayashi A, Kasri NN, Newey SE, Van Aelst L. The Rho-linked mental retardation protein OPHN1 controls synaptic vesicle endocytosis via endophilin A1. Curr Biol. 2009; 19:11331139. [PubMed: 19481455]

10. Sanchez-Barrena MJ, et al. Bin2 is a membrane sculpting N-BAR protein that influences leucocyte podosomes, motility and phagocytosis. PLoS One. 2012; 7:e52401. [PubMed: 23285027]

11. Frost A, Unger VM, De Camilli P. The BAR domain superfamily: membrane-molding macromolecules. Cell. 2009; 137:191-196. [PubMed: 19379681]

12. Rao Y, Haucke V. Membrane shaping by the Bin/amphiphysin/Rvs (BAR) domain protein superfamily. Cell Mol Life Sci. 2011; 68:3983-3993. [PubMed: 21769645]

13. Nishimura T, Morone N, Suetsugu S. Membrane re-modelling by BAR domain superfamily proteins via molecular and non-molecular factors. Biochem Soc Trans. 2018

14. Eichel K, Jullie D, von Zastrow M. beta-Arrestin drives MAP kinase signalling from clathrincoated structures after GPCR dissociation. Nat Cell Biol. 2016; 18:303-310. [PubMed: 26829388]

15. Itoh $\mathrm{T}$, et al. Dynamin and the actin cytoskeleton cooperatively regulate plasma membrane invagination by BAR and F-BAR proteins. Dev Cell. 2005; 9:791-804. [PubMed: 16326391]

16. Shimada A, et al. Curved EFC/F-BAR-domain dimers are joined end to end into a filament for membrane invagination in endocytosis. Cell. 2007; 129:761-772. [PubMed: 17512409]

17. Taylor MJ, Perrais D, Merrifield CJ. A high precision survey of the molecular dynamics of mammalian clathrin-mediated endocytosis. PLoS Biol. 2011; 9:e1000604. [PubMed: 21445324]

18. Tsujita K, Takenawa T, Itoh T. Feedback regulation between plasma membrane tension and membranebending proteins organizes cell polarity during leading edge formation. Nat Cell Biol. 2015; 17:749-758. [PubMed: 25938814]

19. Xiong D, et al. Frequency and amplitude control of cortical oscillations by phosphoinositide waves. Nat Chem Biol. 2016; 12:159-166. [PubMed: 26751515]

20. Pesesse X, et al. The Src homology 2 domain containing inositol 5-phosphatase SHIP2 is recruited to the epidermal growth factor (EGF) receptor and dephosphorylates phosphatidylinositol 3,4,5trisphosphate in EGF-stimulated COS-7 cells. J Biol Chem. 2001; 276:28348-28355. [PubMed: 11349134]

21. Aspenstrom P. A Cdc42 target protein with homology to the non-kinase domain of FER has a potential role in regulating the actin cytoskeleton. Curr Biol. 1997; 7:479-487. [PubMed: 9210375]

22. Ho HY, et al. Toca- 1 mediates Cdc42-dependent actin nucleation by activating the N-WASP-WIP complex. Cell. 2004; 118:203-216. [PubMed: 15260990]

23. Billuart $\mathrm{P}$, et al. Oligophrenin-1 encodes a rhoGAP protein involved in X-linked mental retardation. Nature. 1998; 392:923-926. [PubMed: 9582072]

24. Richnau N, Aspenstrom P. Rich, a rho GTPase-activating protein domain-containing protein involved in signaling by Cdc42 and Rac1. J Biol Chem. 2001; 276:35060-35070. [PubMed: 11431473] 
25. Elbediwy A, et al. Epithelial junction formation requires confinement of Cdc 42 activity by a novel SH3BP1 complex. J Cell Biol. 2012; 198:677-693. [PubMed: 22891260]

26. Bertot L, et al. Quantitative and Statistical Study of the Dynamics of Clathrin-Dependent and Independent Endocytosis Reveal a Differential Role of EndophilinA2. Cell Rep. 2018; 22:15741588. [PubMed: 29425511]

27. Renard HF, et al. Endophilin-A2 functions in membrane scission in clathrin-independent endocytosis. Nature. 2015; 517:493-496. [PubMed: 25517096]

28. Sabharanjak S, Sharma P, Parton RG, Mayor S. GPI-anchored proteins are delivered to recycling endosomes via a distinct cdc42-regulated, clathrin-independent pinocytic pathway. Dev Cell. 2002; 2:411-423. [PubMed: 11970892]

29. Lundmark R, et al. The GTPase-activating protein GRAF1 regulates the CLIC/GEEC endocytic pathway. Curr Biol. 2008; 18:1802-1808. [PubMed: 19036340]

30. Howes MT, et al. Clathrin-independent carriers form a high capacity endocytic sorting system at the leading edge of migrating cells. J Cell Biol. 2010; 190:675-691. [PubMed: 20713605]

31. Sathe M, et al. Small GTPases and BAR domain proteins regulate branched actin polymerisation for clathrin and dynamin-independent endocytosis. Nat Commun. 2018; 91835.

32. Francis MK, et al. Endocytic membrane turnover at the leading edge is driven by a transient interaction between Cdc42 and GRAF1. J Cell Sci. 2015; 128:4183-4195. [PubMed: 26446261]

33. Henne WM, et al. FCHo proteins are nucleators of clathrin-mediated endocytosis. Science. 2010; 328:1281-1284. [PubMed: 20448150]

34. Frost A, et al. Structural basis of membrane invagination by F-BAR domains. Cell. 2008; 132:807817. [PubMed: 18329367]

35. Carmona G, et al. Lamellipodin promotes invasive 3D cancer cell migration via regulated interactions with Ena/VASP and SCAR/WAVE. Oncogene. 2016; 35:5155-5169. [PubMed: 26996666]

36. Hoekstra E, et al. Lipid phosphatase SHIP2 functions as oncogene in colorectal cancer by regulating PKB activation. Oncotarget. 2016; 7:73525-73540. [PubMed: 27716613]

37. Tao Y, et al. SH3-domain binding protein 1 in the tumor microenvironment promotes hepatocellular carcinoma metastasis through WAVE2 pathway. Oncotarget. 2016; 7:18356-18370. [PubMed: 26933917]

38. Baldassarre T, et al. Endophilin A2 Promotes TNBC Cell Invasion and Tumor Metastasis. Mol Cancer Res. 2015; 13:1044-1055. [PubMed: 25784716]

39. Rolland Y, et al. The CDC42-interacting protein 4 controls epithelial cell cohesion and tumor dissemination. Dev Cell. 2014; 30:553-568. [PubMed: 25203208]

40. Endris V, et al. SrGAP3 interacts with lamellipodin at the cell membrane and regulates Racdependent cellular protrusions. J Cell Sci. 2011; 124:3941-3955. [PubMed: 22159416]

41. Krause M, et al. Lamellipodin, an Ena/VASP ligand, is implicated in the regulation of lamellipodial dynamics. Dev Cell. 2004; 7:571-583. [PubMed: 15469845]

42. Doherty GJ, et al. The endocytic protein GRAF1 is directed to cell-matrix adhesion sites and regulates cell spreading. Mol Biol Cell. 2011; 22:4380-4389. [PubMed: 21965292]

43. Vizcaíno JA, et al. 2016 update of the PRIDE database and related tools. Nucleuc Acids Res. 2016; 44:D447-456.

Nat Cell Biol. Author manuscript; available in PMC 2019 March 01. 

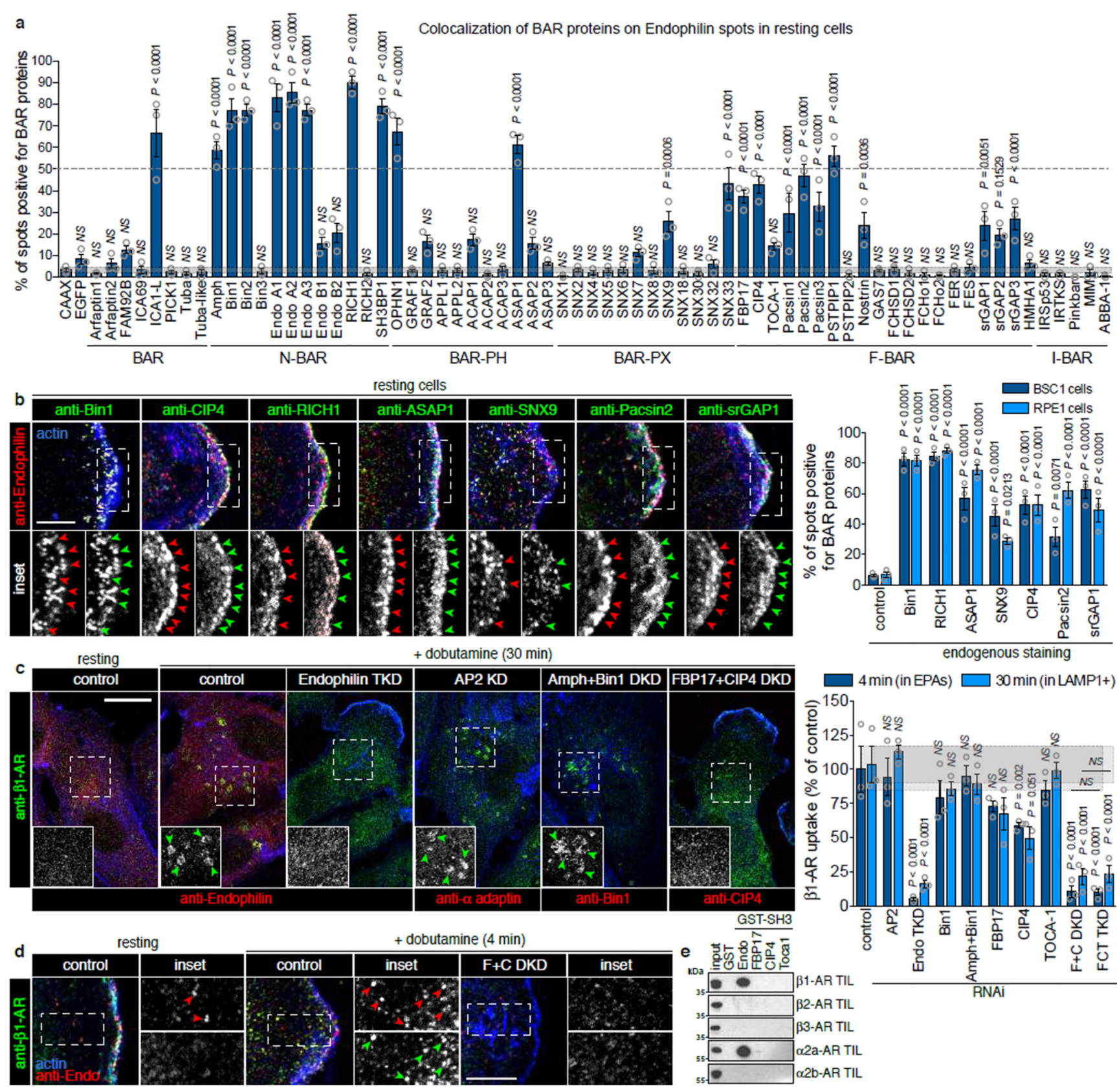

Figure 1. FBP17 and CIP4 colocalize with Endophilin and mediate $\beta 1-A R$ uptake.

a, Colocalization of named EGFP-tagged BAR proteins on endogenous Endophilin spots in

BSC1 cells. Histograms show the mean \pm SEM from three independent biological

experiments ( $n>150$ puncta per construct). b, Colocalization of named endogenous BAR proteins on Endophilin spots at the leading edge of resting BSC1 cells. Histograms show the mean \pm SEM from three independent biological experiments ( $n=150$ puncta per condition).

c, Intracellular accumulation of $\beta 1$ adrenergic receptor ( $\beta 1-\mathrm{AR})$ in Endophilin triple knocked-down (TKD), AP2 knocked-down (KD), Amphiphysin and Bin1 double knocked-

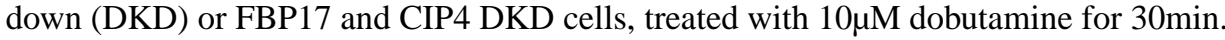


Counterstaining of the targeted proteins (red) validated the knock-downs in the cells imaged. Histograms show the mean \pm SEM from three independent biological experiments ( $n=30$ cells per condition), normalized to control cells. $\mathbf{d}, \beta 1$-AR uptake into FEME carriers (cytoplasmic Endophilin-positive assemblies) following $4 \mathrm{~min}$ addition of $10 \mu \mathrm{M}$ dobutamine in cells depleted for FBP17 and CIP4 (F+C DKD) or not. Histograms show the mean \pm SEM from three independent biological experiments ( $n=30$ cells per condition). e, Pull-down using GST or GST-SH3 domains of Endophilin A2, FBP17, CIP4 and TOCA-1 and cell extracts expressing EGFP-tagged third intracellular loops (TIL) of $\beta 1-, \beta 2-, \beta 3-$, a $2 \mathrm{a}-$ or a 2b-adrenergic receptors. Inputs correspond to 1 to $5 \%$ of the cell extracts. Unprocessed original scans are provided in Supplementary Fig. 6. All images are representative of at least 10 captures taken from three independent biological experiments for each condition. All experiments were repeated independently at least three times with similar results. Statistical analysis was performed by one-way ANOVA (a) or two-way ANOVA (b,c); $N S$, non significant $\mathrm{P}>0.99$. Statistical source data are provided in Supplementary Table 2. Scale bars, $10(\mathbf{c})$ and $5 \mu \mathrm{m}(\mathbf{b}, \mathbf{d})$. 

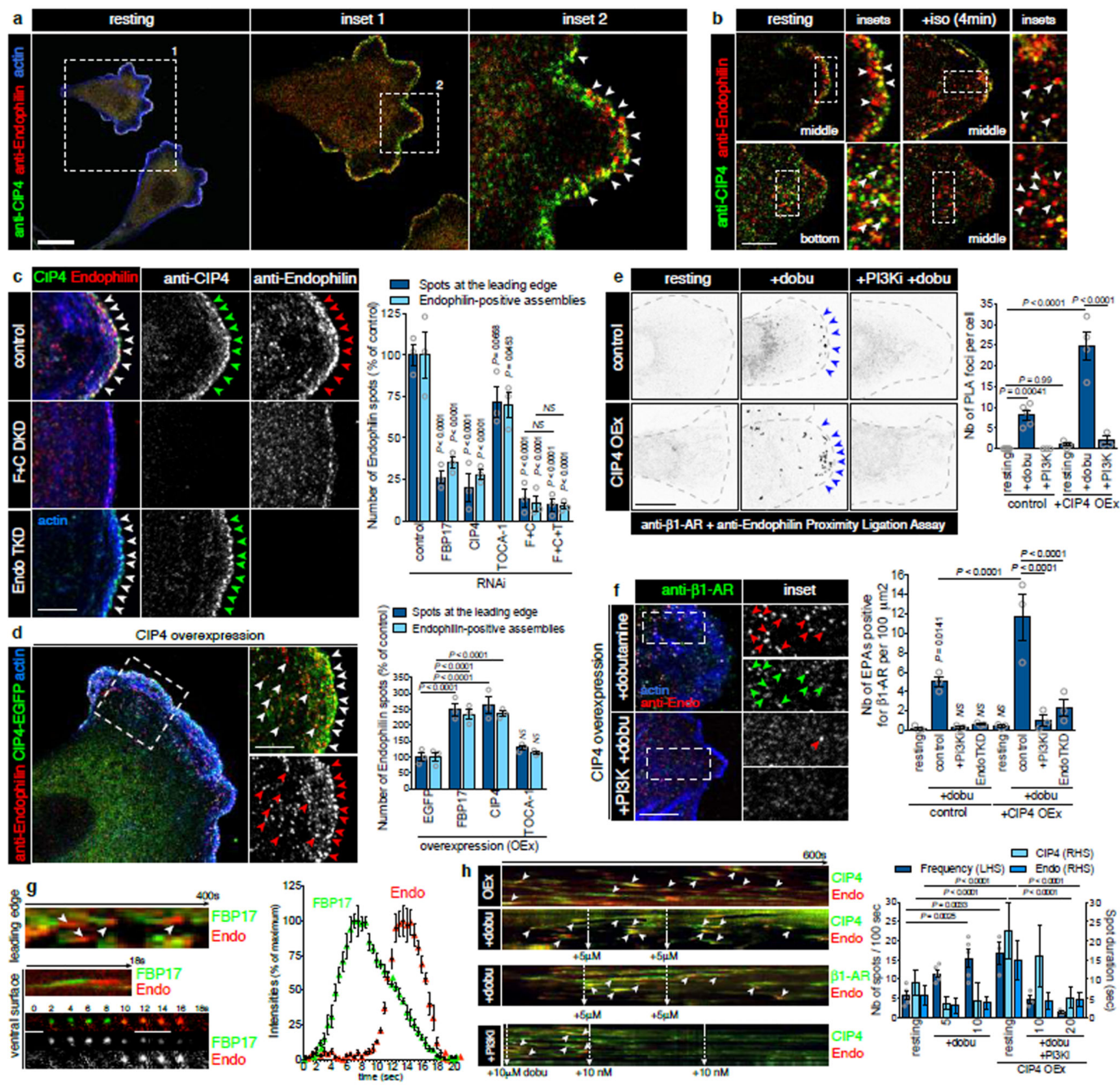

Figure 2. FBP17 and CIP4 prime cells for FEME.

a, colocalization of endogenous CIP4 and Endophilin in resting BSC1 cells. b,

Colocalization of CIP4 on Endophilin spots at the plasma membrane but not on FEME

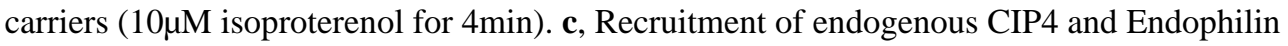
in resting cells depleted or not for FBP17 and CIP4 (F+C DKD) or Endophilin (Endo TKD). Histograms show the mean \pm SEM from three independent biological experiments $(n=150$ cells per condition), normalized to control. d, Endogenous Endophilin cell surface spots and FEME carriers in cells overexpressing CIP4-EGFP (CIP4 OEx). Histograms show the mean \pm SEM from three independent biological experiments ( $n>30$ cells per condition), 
normalized to control. e, Proximity Ligation Assays between endogenous $\beta 1-A R$ and Endophilin in CIP4 OEx or control cells. Cells were pre-treated with 20nM GDC-0941 (PI3Ki) for $5 \mathrm{~min}$, before stimulation with $10 \mu \mathrm{M}$ dobutamine for $4 \mathrm{~min}$, as indicated. Histograms show the mean \pm SEM from three independent biological experiments $(n>30$ cells per condition). f, $\beta 1$-AR uptake into FEME carriers in cells overexpressing CIP4-Myc and treated as in e. Histograms show the mean \pm SEM from three independent biological experiments ( $n=30$ cells per condition). $\mathbf{g}$, Kymographs and till views from leading edge or ventral surface of resting cells expressing low levels of FBP17-EGFP and EndophilinA2RFP and imaged at $0.5 \mathrm{~Hz}$ (top) and $2 \mathrm{~Hz}$ (middle). Right, percentage of maximum signals (means \pm SEM, $n=50$ spots from three independent biological experiments). $\mathbf{h}$, Kymographs from cells expressing low levels of $\beta 1$-AR-EGFP or EndophilinA2-RFP and OEx or not CIP4-EGFP, treated with dobutamine and GDC-0941 as indicated and imaged at 2Hz. Kymographs are representative of 5 captures from three independent biological experiments. Histograms show the mean \pm SEM from three independent biological experiments ( $n=5$ cells per condition). All images are representative of at least 10 captures taken from three independent biological experiments for each condition. All experiments were repeated independently at least three times with similar results. Statistical analysis was performed by one-way ANOVA (e,f) or two-way ANOVA $(\mathbf{c}, \mathbf{d}, \mathbf{h}) ; N S$, non significant $\mathrm{P}>0.99$. Statistical source data are provided in Supplementary Table 2. Scale bars, 20 (a) and $5 \mu \mathrm{m}(\mathbf{b}, \mathbf{c}, \mathbf{d}, \mathbf{f})$. 

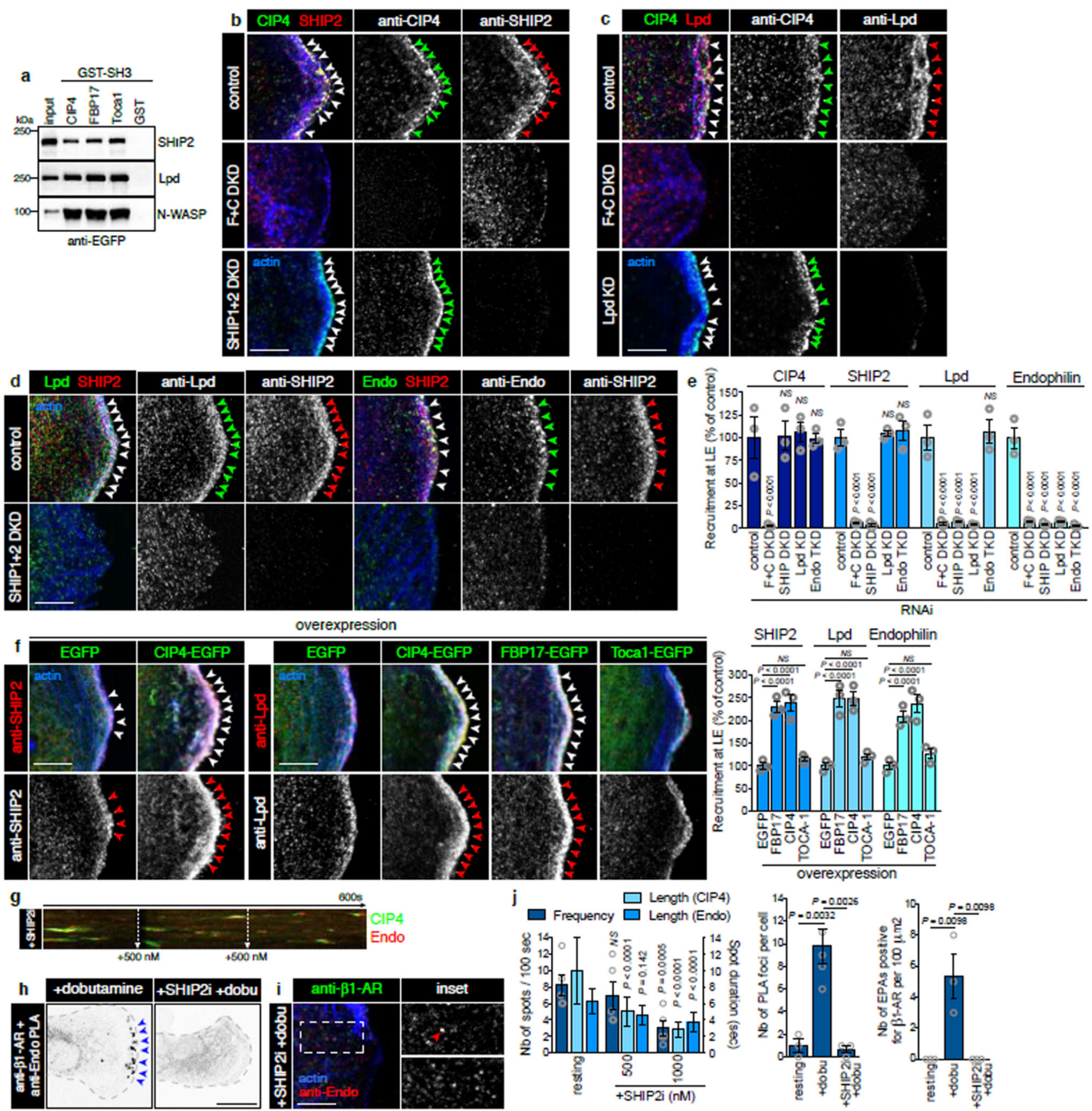

Figure 3. FBP17 and CIP4 recruit SHIP2 and Lpd.

a, Pull-down experiments with GST-tagged SH3 domains of the indicated proteins and EGFP-tagged SHIP2, Lpd or N-WASP (positive control). Inputs correspond to 1 to $5 \%$ of the cell extracts. Unprocessed original scans are provided in Supplementary Fig. 6. b-e, Recruitment of endogenous CIP4, SHIP2, Lpd or Endophilin in resting BSC1 cells depleted or not for FBP17 and CIP4 (F+C DKD), SHIP (SHIP1+2 DKD) or Lpd, as indicated.

Histograms show the mean \pm SEM from three independent biological experiments ( $n=30$ cells per condition), normalized to control. f, Recruitment endogenous SHIP2 or Lpd at the 
leading edge of resting cells overexpressing the indicated constructs. Histograms show the mean \pm SEM from three independent biological experiments ( $n=30$ cells per condition), normalized to control. g, Kymograph from a cell expressing low levels of CIP4-EGFP and EndophilinA2-RFP, treated with AS19499490 (SHIP2i) as indicated and imaged at 2Hz. The kymograph is representative of 9 captures from three independent biological experiments. $\mathbf{h}$, Proximity Ligation Assays between endogenous $\beta 1$-AR and Endophilin in cells pre-treated

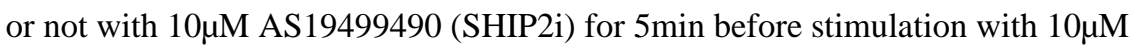
dobutamine for 4min, as indicated. i, $\beta 1$-AR uptake into FEME carriers (cytoplasmic Endophilin-positive assemblies) in cells treated as in $\mathbf{h}$. $\mathbf{j}$, Histograms show the mean \pm SEM (left and middle, $n=30$ cells per condition, right, $n=5$ cells per condition) of cells treated as in $\mathbf{g - i}$, respectively. All images are representative of at least 10 captures taken from three independent biological experiments. All experiments were repeated independently at least three times with similar results. Statistical analysis was performed by one-way ANOVA ( $\mathbf{j}$, middle and right) or two-way ANOVA (e,f,j left); $N S$, non significant $\mathrm{P}>0.99$. Statistical source data are provided in Supplementary Table 2. Scale bars, 20 (h) and $5 \mu \mathrm{m}(\mathbf{b}, \mathbf{c}, \mathbf{d}, \mathbf{f}, \mathbf{i})$. 

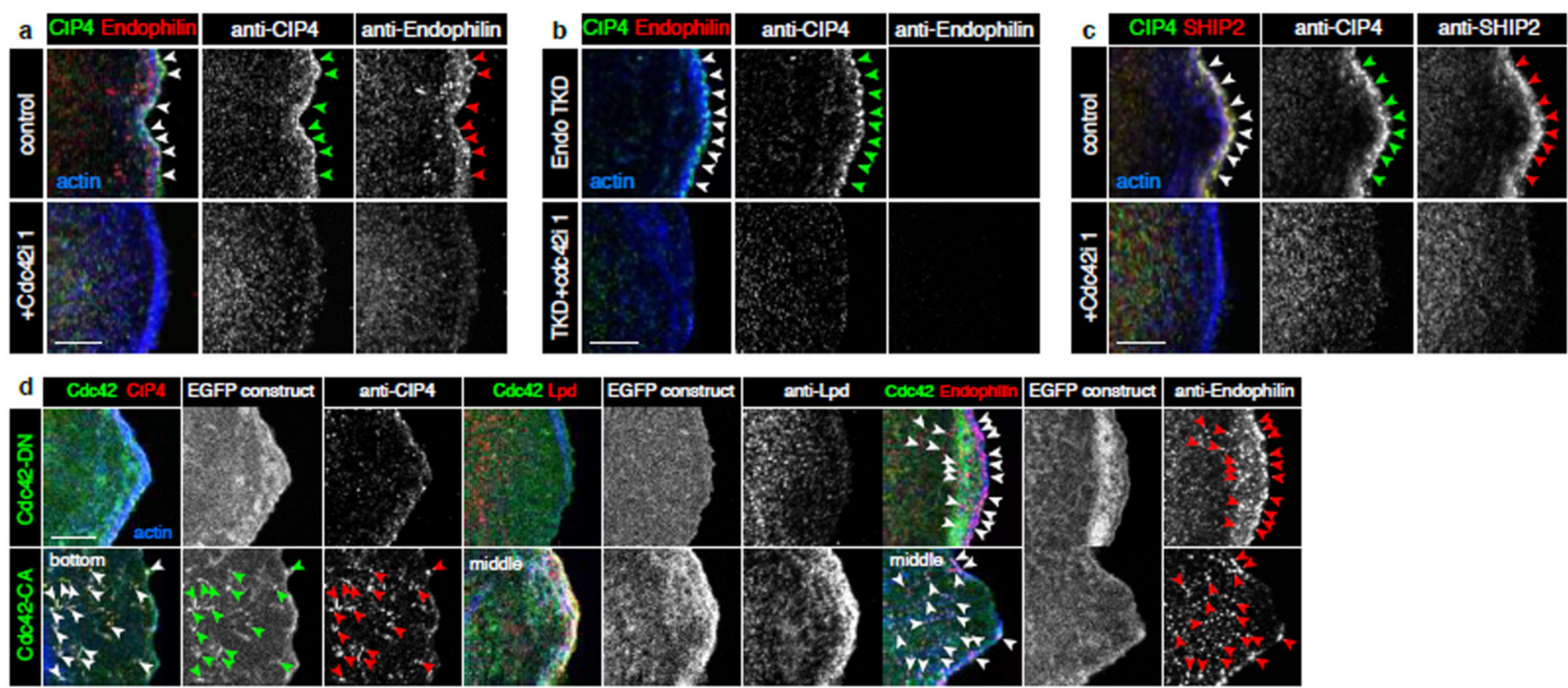

e $\square$ Spots at the leading edge

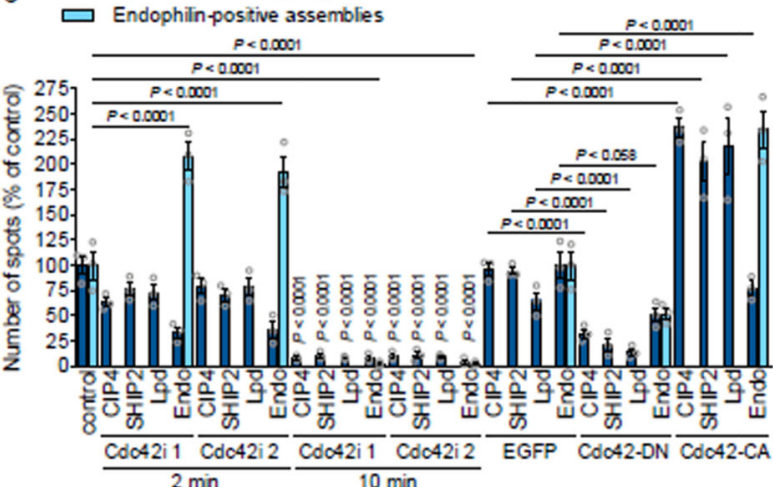

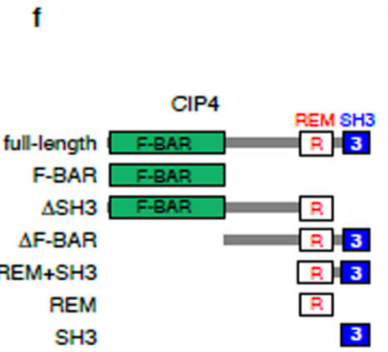

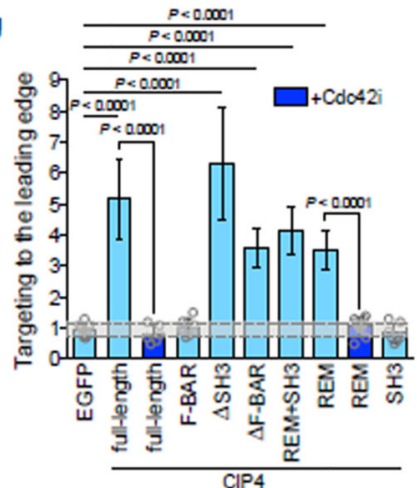

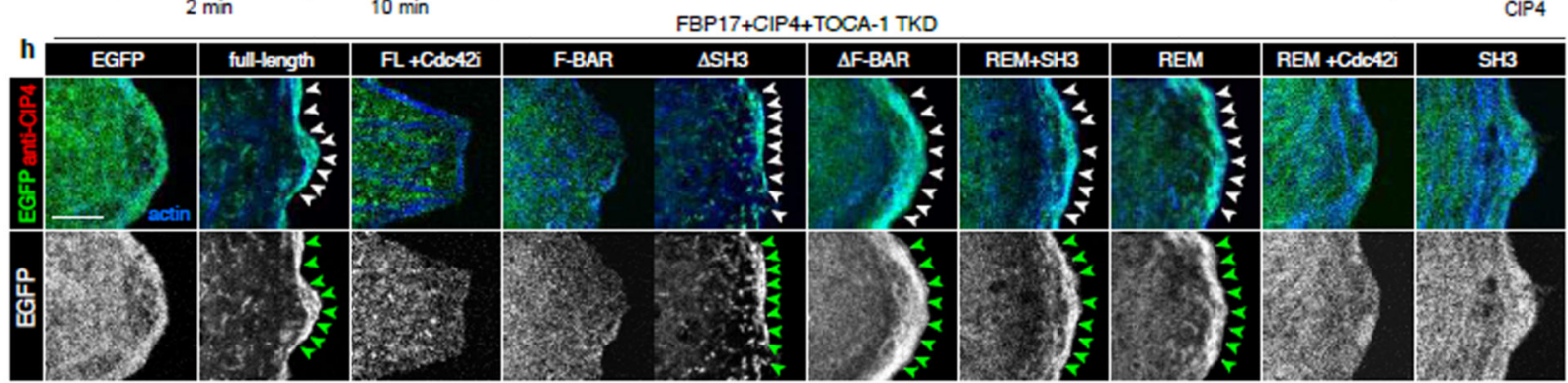

Figure 4. GTP-loaded Cdc42 recruits FBP17 and CIP4 to the plasma membrane.

a-c, Recruitment of endogenous CIP4, SHIP2, or Endophilin in resting BSC1 cells depleted

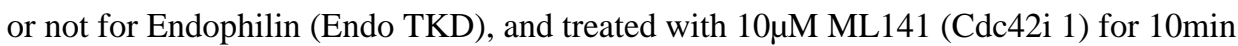
with as indicated. Images are representative of 10 captures from three independent biological experiments for each condition. d, Recruitment of endogenous CIP4, Lpd or Endophilin in resting cells overexpressing EGFP-tagged dominant negative (Cdc42-DN, T17N mutant) or constitutively active (Cdc42-CA, Q61L mutant) versions of Cdc42, as indicated. Focal planes were located at the bottom membrane or in the middle of the cells, as indicated. Images are representative of 6 captures from three independent biological experiments for each condition. e, Histograms show the mean \pm SEM from three 
independent biological experiments ( $n=30$ cells per condition), treated as in a-d and normalized to the respective controls. Secramine (Cdc42i 2) was used at $10 \mu \mathrm{M}$ for the indicated time). $\mathbf{f}$, scheme of the EGFP-tagged full-length or truncated versions of CIP4 used. $\mathbf{g}, \mathbf{h}$, Recruitment of EGFP-tagged full-length or truncated versions of CIP4 in cells depleted for endogenous FBP17, CIP4 and TOCA1 (TKD) and treated or not with 10 $\mu \mathrm{M}$ ML141 for 10min (+Cdc42i), as indicated. Cells were immunostained for endogenous CIP4 (red) to control for the depletion in the cells imaged. Images are representative of at least 6 captures taken from three independent biological experiments for each condition.

Histograms show the mean \pm SEM ( $n>6$ cells per condition). All experiments were repeated at least three times with similar results. Statistical analysis was performed by one-way ANOVA (g) or two-way ANOVA (e). Statistical source data are provided in Supplementary Table 2. Scale bars, $5 \mu \mathrm{m}$. 


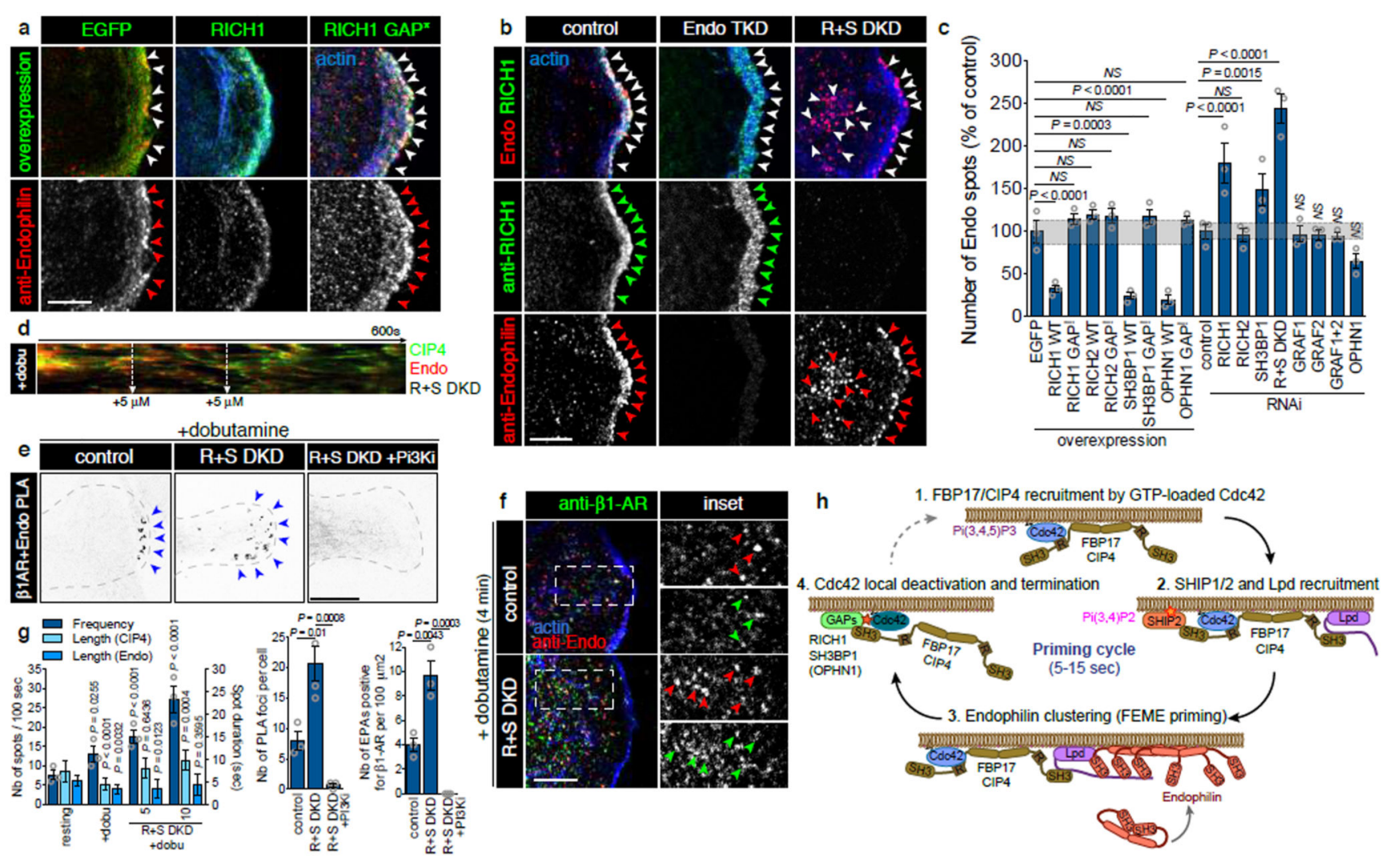

Figure 5. Local recruitment of Cdc42GAPs terminates the priming cycle.

a, Recruitment of endogenous Endophilin in resting BSC1 cells overexpressing wild-type EGFP-tagged RICH1 or R288A mutant (GAP*), as indicated. Images are representative of 10 captures from three independent biological experiments. b, Recruitment of endogenous Endophilin and RICH1 in resting cells depleted or not for Endophilin (Endo TKD) or RICH1 and SH3BP1 (R+S DKD). Images are representative of 10 captures from three independent biological experiments. c, Histograms show the mean \pm SEM from three independent biological experiments ( $n=30$ cells per condition) treated as indicated and normalized to the respective controls. d, Kymograph from a $\mathrm{R}+\mathrm{S}$ DKD cell expressing low levels of CIP4-EGFP and EndophilinA2-RFP and stimulated with dobutamine at the indicated times. The kymograph is representative of 5 captures from three independent biological experiments. e, Proximity Ligation Assays between endogenous $\beta 1-\mathrm{AR}$ and Endophilin in cells R+S DKD depleted pre-treated or not with 20nM GDC-0941 (PI3Ki) for $5 \mathrm{~min}$ before stimulation with $10 \mu \mathrm{M}$ dobutamine for $4 \mathrm{~min}$, as indicated. $\mathbf{f}, \beta 1$-AR uptake into FEME carriers (cytoplasmic Endophilin-positive assemblies) in cells treated as in e. $\mathbf{g}$, Histograms show the mean \pm SEM (left and middle, $n=30$ cells per condition, right, $n=3$ cells per condition) of cells treated as in d-f, respectively. (h) Model summarizing the priming cycle of FEME in resting cells: Step 1, active GTP-loaded Cdc42 recruits FBP17 and CIP4 through their REM domains. Step 2, FBP17 and CIP4 cluster 5'-phosphatases SHIP1 and 2 as well as Lpd through their SH3 domains. Lpd is further stabilized by $\mathrm{Pi}(3,4) \mathrm{P}_{2}$ locally produced by SHIP1/2, Step 3, Endophilin is recruited and concentrated by Lpd. From there, pre-enriched Endophilin mediates prompt FEME carrier formation upon 
cargo activation. In absence of cargo activation, the FEME priming complex disassembled (Step 4), upon local Cdc42 deactivation by the GAPs RICH1 and SH3BP1. All experiments were repeated at least three times with similar results. Statistical analysis was performed by one-way ANOVA (c, $\mathbf{g}$ middle and right) or two-way ANOVA (g left); $N S$, non significant $\mathrm{P}>0.99$. Statistical source data are provided in Supplementary Table 2. Scale bars, $20(\mathbf{e})$ and $5 \mu \mathrm{m}(\mathbf{a}, \mathbf{b}, \mathbf{f})$. 